\title{
Impact of lung disease on respiratory impedance in young children with cystic fibrosis
}

\author{
Kathryn A. Ramsey ${ }^{1,2}$, Sarath C. Ranganathan ${ }^{3,4,5}$, Catherine L. Gangell ${ }^{1,6}$, \\ Lidija Turkovic ${ }^{1}$, Judy Park ${ }^{1}$, Billy Skoric ${ }^{3,4}$, Stephen M. Stick ${ }^{1,7}$, Peter D. Sly ${ }^{6}$ \\ and Graham L. Hall ${ }^{1}$, on behalf of AREST CF ${ }^{8}$
}

\begin{abstract}
Affiliations: ${ }^{1}$ Telethon Kids Institute, University of Western Australia, Subiaco, Australia. ${ }^{2}$ Cystic Fibrosis Research and Treatment Centre, University of North Carolina at Chapel Hill, Chapel Hill, NC, USA. ${ }^{3}$ Murdoch Childrens Research Institute, Parkville, Australia. "Department of Respiratory Medicine, Royal Children's Hospital, Parkville, Australia. ${ }^{5}$ Department of Paediatrics, University of Melbourne, Parkville, Australia. ${ }^{6}$ Queensland Children's Medical Research Institute, The University of Queensland, Herston, Australia. ${ }^{7}$ Department of Respiratory Medicine, Princess Margaret Hospital for Children, Subiaco, Australia. ${ }^{8}$ Details of the full membership of the Australian Respiratory Early Surveillance Team for Cystic Fibrosis (AREST CF) are available at www.arestcf.org/our-people.
\end{abstract}

Correspondence: Graham Hall, Paediatric Respiratory Physiology, Telethon Kids Institute, 100 Roberts Road, Subiaco, WA 6008 Australia. E-mail: Graham.Halldtelethonkids.org.au

ABSTRACT This study aimed to evaluate the ability of the forced oscillation technique (FOT) to detect underlying lung disease in preschool children with cystic fibrosis (CF) diagnosed following newborn screening.

184 children (aged 3-6 years) with CF underwent lung function testing on 422 occasions using the FOT to assess respiratory resistance and reactance at the time of their annual bronchoalveolar lavage collection and chest computed tomography scan. We examined associations between FOT outcomes and the presence and progression of respiratory inflammation, infection and structural lung disease.

Children with CF who had pronounced respiratory disease, including free neutrophil elastase activity, infection with pro-inflammatory pathogens and structural lung abnormalities had similar FOT outcomes to those children without detectable lung disease. In addition, the progression of lung disease over 1 year was not associated with worsening FOT outcomes.

We conclude that the forced oscillation technique is relatively insensitive to detect underlying lung disease in preschool children with CF. However, FOT may still be of value in improving our understanding of the physiological changes associated with early CF lung disease.

@ERSpublications

Forced oscillation technique is insensitive in detecting lung disease in preschool children with cystic fibrosis http://ow.ly/R9rSU

Received: Jan 282015 | Accepted after revision: July 142015 | First published online: Sept 242015

Support statement: Funding for the Australian Respiratory Early Surveillance Team for Cystic Fibrosis (AREST CF) programme was provided by the Cystic Fibrosis Foundation Therapeutics (SLY04A0 and STICK09A0) and the National Health and Medical Research Council of Australia (NHMRC) (APP513730 and Centre of Research Excellence \#1000896). G. Hall (APP1025550) and K. Ramsey (APP1088389) are NHMRC fellows. Funding information for this article has been deposited with FundRef.

Conflict of interest: None declared.

Copyright OERS 2015 


\section{Introduction}

Lung disease begins in infancy in individuals with cystic fibrosis (CF) and progresses rapidly during the preschool years $[1,2]$. With the recent development of disease-modifying treatments for $\mathrm{CF}$, there is a need for noninvasive techniques to monitor disease development and progression in young children [3, 4]. While lung function tests offer an alternative to invasive surveillance, studies correlating disease outcomes and respiratory function have predominantly been limited to those using spirometry [5-7] or techniques that require sedation [8-14], and there have been relatively few studies in preschool-aged children [8, 15-20].

The forced oscillation technique (FOT) is a promising lung function test that can measure the respiratory impedance of the lung in children as young as 2 years of age $[17,21,22]$. This technique is well suited to measurements in young children as it requires only tidal breathing and minimal cooperation from the child, and can be completed within 5-15 min. The FOT has been reported to be sensitive to detect respiratory function abnormalities in young children with $\mathrm{CF}[8,19,23-25]$, asthma $[19,23,25]$ and neonatal chronic lung disease $[22,26,27]$. Evidence of the clinical utility of the FOT in detecting early CF lung disease is not available [28].

The ability of lung function tests to detect early lung disease, namely inflammation, infection and structural lung abnormalities in young children with CF is not well reported. There are only limited studies investigating associations between inflammatory markers and measurements of lung function in preschool children $[14,29]$. Associations with lung function measurements and infections have been generally limited to Pseudomonas aeruginosa infections, with studies reporting inconsistent results [11, 14, $15,17,19,20,29]$. Our group has previously demonstrated that FOT is sensitive to changes in respiratory symptoms in young children with CF [24]. However, relationships between markers of lung disease, assessed using bronchoalveolar lavage (BAL) and chest computed tomography (CT) and FOT outcomes have yet to be examined. The present study was undertaken to test the hypothesis that lung function measured by FOT would be impaired in the presence of infection, inflammation and structural lung disease in young children with CF.

\section{Methods}

Children diagnosed with CF following newborn screening were recruited from the CF clinics at Princess Margaret Hospital for Children (Perth, Australia) and the Royal Children's Hospital (Melbourne, Australia) and enrolled into the Australian Respiratory Early Surveillance Team for Cystic Fibrosis (AREST $\mathrm{CF}$ ) early surveillance programme. The programme includes assessment soon after diagnosis and then annually until the child reaches the age of 6 years. This programme has been described in full elsewhere [1]. These annual assessments include a bronchoscopy with BAL fluid collection, chest CT and age-appropriate lung function testing. In preschool children, between the ages of 3 and 6 years, the FOT was performed. This study included data from annual assessments performed between October 2002 and April 2014. The study was approved by the ethics committee of each institution and parents consented to each aspect of the study separately.

\section{Lung function using FOT}

Respiratory function was performed on the morning of, or the day prior to, the child's annual assessment according to international preschool lung function testing guidelines [30]. Measurements of respiratory system input impedance were obtained using a commercial device (I2M; Chess Medical, Ghent, Belgium). Respiratory impedance $(\mathrm{Zrs})$ measurements in which three or more frequencies had a coherence $<0.95$ were excluded. Three to five acceptable $Z$ rs measurements were obtained for each child and the mean values of the resistance $(R \mathrm{rs})$ and reactance $(X \mathrm{rs})$ of the respiratory system at each frequency were reported. The frequency dependence ( $f_{\text {dep }}$ ) of $R$ rs between $4 \mathrm{~Hz}$ and $24 \mathrm{~Hz}$ and the area under the reactance curve $(A \mathrm{x})$, using the trapezium rule to approximate the integral, between $6 \mathrm{~Hz}$ and the resonant frequency were calculated.

\section{Bronchoalveolar lavage}

A bronchoscopy was performed after the CT scan, during which BAL fluid was collected. The right middle lobe was lavaged with three aliquots of saline $\left(1 \mathrm{~mL} \cdot \mathrm{kg}^{-1}\right.$ body weight $)$ and an additional aliquot lavaged into the lingula or the most affected lobe identified on CT $[1,31]$. The first aliquot of BAL fluid was processed for the detection of bacteria, viruses and fungi using standard culture techniques. An infection with a bacterial or fungal load of $\geqslant 10^{5} \mathrm{cfu} \cdot \mathrm{mL}^{-1}$ (excluding mixed oral flora) was considered significant. Bacterial pathogens known to elicit a pro-inflammatory response when isolated from the lower respiratory tract (P. aeruginosa, Staphylococcus aureus, Haemophilus influenzae, Streptococcus pneumoniae and Aspergillus species) were described as pro-inflammatory pathogens [31]. Subsequent aliquots were pooled for the quantification of pulmonary inflammation which included total and differential cell counts, interleukin (IL)-8 and measurement of free neutrophil elastase activity (lower limit of detection $\left.200 \mathrm{ng} \cdot \mathrm{mL}^{-1}\right)[1]$. 
Chest CT

Chest CT scans in preschool children were performed prior to BAL collection under intravenous general anaesthetic. Volumetric helical or limited slice inspiratory chest CT scans were obtained at a positive airway opening pressure of $25 \mathrm{cmH}_{2} \mathrm{O}$. Limited slice expiratory chest CT scans were obtained at functional residual capacity. The CT images were scored for the presence of structural lung disease using a simplified CF CT scoring method $[1,2]$. Scans were assessed by lobe (with the lingula treated as a separate lobe) and each lobe was scored for the presence and extent of bronchiectasis (bronchus-to-artery ratio $>1$ on inspiratory scan) and air trapping (expiratory scan). Details of the scanners and settings used have been published previously [32].

\section{Respiratory symptoms and clinical data}

Height and weight $\mathrm{z}$-scores were calculated on the basis of World Health Organization growth standards $[33,34]$. A standardised respiratory symptoms questionnaire was conducted on the morning of the BAL to gain information on parentally reported symptoms (cough, upper respiratory tract infection and sputum production) and current medication use. A respiratory clinician performed a physical examination of the infant and reported the presence or absence of wheeze, crackles or respiratory tract infection.

\section{Statistical analysis}

Resistance, $X \mathrm{rs}$, resonant frequency of respiratory reactance ( $f_{\text {res }}$ ) and $A \mathrm{x}$ data were expressed as z-scores determined from reference ranges derived from an international multicentre collation of FOT outcomes [17]. Frequency dependence is independent of demographic factors and absolute values were used [17]. Univariate mixed effects models with random subject intercepts were used to examine the association between inflammation, infection and structural lung disease with lung function. The outcome variables in our models were the lung function measurements and the explanatory variables were inflammation markers, infection status and CT scores that indicate presence and extent of structural lung disease. Initially cross-sectional analyses were performed to investigate these relationships followed by longitudinal analyses of differences in FOT outcomes in two adjacent visits by the same subject occurrring between 6 and 18 months. Since the inflammatory markers were positively skewed, natural logarithmic transformations were applied to the data to satisfy normality. All analyses were performed using Stata (version 13.0; StataCorp, College Station, TX, USA) and presented as coefficients, 95\% CI and p-values.

\section{Results}

FOT data matched to either BAL or chest CT were collected at annual review on 422 occasions in 184 children aged 3-6 years between October 2002 and April 2014 (table 1). At the annual review visits 50\% of children had current respiratory symptoms, 33\% had free neutrophil elastase activity and $48 \%$ had a significant lower respiratory tract infection. The most common pathogen detected was S. aureus (14\%), followed by $H$. influenzae (13\%), P. aeruginosa (10\%), Aspergillus species (9\%) and S. pneumoniae (3\%). In the annual review visits with matched chest CT, bronchiectasis and air trapping were detected in $70 \%$ and $74 \%$ of scans, respectively.

\section{Inflammation}

In young children with CF, neutrophil count and IL-8 count were associated with lower $f$ dep $(-0.007$, $-0.011--0.003 ; \mathrm{p}<0.001$ and $-0.007,-0.012--0.003 ; \mathrm{p}=0.002$, respectively) and $\mathrm{Xrs}$ at $8 \mathrm{~Hz}\left(X_{\mathrm{rs}} 8\right) \mathrm{z}$-score $(-0.073,-0.119--0.027 ; \mathrm{p}=0.002$ and $-0.078,-0.137--0.019 ; \mathrm{p}=0.010$, respectively) (tables 2 and 3$)$. Longitudinally, neutrophil count was associated with an increase in fres z-score (0.091, 0.001-0.162; $\mathrm{p}=0.022$ ) but was not associated in any other changes in FOT outcomes (tables 4 and 5). There were no associations between the presence of neutrophil elastase and any FOT outcomes in either cross-sectional or longitudinal analyses.

\section{Infection}

As a group, children infected with any respiratory pathogen or a pro-inflammatory pathogen at annual review had the same FOT outcomes as uninfected children, and infection with a pathogen was not associated with a change in FOT outcomes over 1 year. However, when we examined the pathogens individually we found that children infected with $H$. influenzae had higher $R$ rs at $8 \mathrm{~Hz}$ z-scores $(0.389$, $0.051-0.727 ; \mathrm{p}=0.024)$, lower $f_{\text {dep }}(-0.053,-0.092--0.014 ; \mathrm{p}=0.008)$, lower $X \mathrm{rs8} \mathrm{Z}$-scores $(-0.643,-1.143-$ $-0.142 ; \mathrm{p}=0.012)$ and higher fres $\mathrm{z}$-scores $(0.475,0.012-0.937 ; \mathrm{p}=0.044)$ compared with those without a respiratory infection. In addition, infection with $H$. influenzae was associated with an increase in $A \mathrm{x}$ $\mathrm{z}$-score over 1 year $(1.087,0.261-1.913 ; \mathrm{p}=0.010)$. There were no other associations between other individual respiratory pathogens and FOT outcomes in either cross-sectional or longitudinal analyses. 


\section{TABLE 1 Characteristics of study participants}

Subjects
Visits
Age years mean (range)
Sex male:female (\% male)
Height z-score
Weight z-score
Clinical status
Phe508del/Phe508del
Phe508del/other
Severe genotype
Current respiratory symptoms
Inflammatory status
Presence of IL-8
Presence of neutrophil elastase
Infection status
No infection
Mixed oral flora only
Significant infection
Multiple pathogens
Staphylococcus aureus
Haemophilus influenzae
Pseudomonas aeruginosa
Aspergillus species
Streptococcus pneumoniae
Structural lung disease
Bronchiectasis
Air trapping

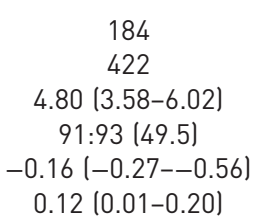

$20.7(34 / 164)$

$68.9(113 / 164)$

$90.9(149 / 164)$

$50.0(142 / 284)$

$94.1(382 / 406)$

$32.7(133 / 407)$

$21.3(90 / 422)$

$19.9(84 / 422)$

$47.9(202 / 422)$

$15.9(67 / 422)$

$14.0(59 / 422)$

$13.3(55 / 422)$

$9.5(40 / 422)$

$8.6(34 / 422)$

$2.8(12 / 422)$

$70.2(146 / 208)$

$73.7(73 / 99)$

Data are presented as $\mathrm{n}$, mean $(95 \% \mathrm{Cl})$ or $\%(\mathrm{n} / \mathrm{N})$, unless otherwise stated. IL: interleukin. Height and weight z-scores were calculated on the basis of World Health Organization growth standards [33, 34].

Structural lung disease

Children with air trapping detected on chest CT at annual review had lower $f_{\text {dep }}(-0.007,-0.014--0.001$; $\mathrm{p}=0.045)$ and lower $X \mathrm{rs8} \mathrm{z}$-scores $(-0.104,-0.193--0.016 ; \mathrm{p}=0.021)$ than those without air trapping. Longitudinally, the presence of air trapping was associated with a decrease in $f$ dep $(-0.017,-0.030--0.005$; $\mathrm{p}=0.008)$ and $X \mathrm{rs} 8 \mathrm{z}$-score $(-0.182,-0.317-0.046 ; \mathrm{p}=0.009)$ over 1 year. In the cross-sectional analysis, children with bronchiectasis had the same FOT outcomes as those without bronchiectasis detected at

TABLE 2 Cross-sectional analysis of respiratory resistance outcomes and cystic fibrosis (CF) lung disease parameters

\begin{tabular}{|c|c|c|c|c|}
\hline & Rrs8 z-score & p-value & fdep & p-value \\
\hline Severe genotype & $-0.396(-0.845-0.053)$ & 0.084 & $0.003(-0.039-0.046)$ & 0.886 \\
\hline Current respiratory symptoms & $-0.163(-0.519-0.193)$ & 0.370 & $0.011(-0.026-0.049)$ & 0.560 \\
\hline Neutrophil count & $0.009(-0.024-0.042)$ & 0.588 & $-0.007(-0.011--0.003)$ & $<0.001$ \\
\hline Presence of neutrophil elastase & $-0.110(-0.378-0.158)$ & 0.421 & $-0.004(-0.019-0.028)$ & 0.726 \\
\hline IL-8 count & $0.007(-0.035-0.050)$ & 0.730 & $-0.007(-0.012--0.003)$ & 0.002 \\
\hline Significant infection & $0.112(-0.132-0.356)$ & 0.368 & $-0.018(-0.047-0.011)$ & 0.231 \\
\hline Proinflammatory pathogens & $0.133(-0.107-0.373)$ & 0.278 & $-0.027(-0.057-0.002)$ & 0.072 \\
\hline Staphylococcus aureus & $0.056(-0.278-0.390)$ & 0.742 & $-0.035(-0.074-0.003)$ & 0.073 \\
\hline Haemophilus influenzae & $0.389(0.051-0.727)$ & 0.024 & $-0.053(-0.092--0.014)$ & 0.008 \\
\hline Pseudomonas aeruginosa & $0.015(-0.405-0.418)$ & 0.975 & $-0.047(-0.095-0.001)$ & 0.054 \\
\hline Aspergillus spp. & $0.017(-0.335-0.370)$ & 0.923 & $0.001(-0.037-0.040)$ & 0.941 \\
\hline Multiple infection & $-0.202(-0.523-0.118)$ & 0.216 & $0.037(0.005-0.069)$ & 0.023 \\
\hline Bronchiectasis score & $-0.015(-0.051-0.021)$ & 0.420 & $-0.003(-0.007-0.001)$ & 0.112 \\
\hline Air-trapping score & $0.032(-0.031-0.10)$ & 0.324 & $-0.007(-0.014--0.001)$ & 0.045 \\
\hline
\end{tabular}

Data are presented as mean $(95 \% \mathrm{Cl}$ ) difference between children with $\mathrm{CF}$ described by the variable compared with those not described by the variable, unless otherwise stated. Statistically significant $(p<0.05)$ changes are shown in bold. Rrs8: respiratory resistance at $8 \mathrm{~Hz}$; fdep: frequency dependence of respiratory resistance; IL: interleukin. 
TABLE 3 Cross-sectional analysis of respiratory reactance outcomes and cystic fibrosis (CF) lung disease parameters

\begin{tabular}{lcccccc} 
& Xrs8 & p-value & fres & p-value & Ax & p-value \\
\hline Severe genotype & $0.235(-0.325-0.794)$ & 0.411 & $-\mathbf{0 . 5 6 0}(-\mathbf{1 . 1 2 8 - - 0 . 0 6 5 )}$ & $\mathbf{0 . 0 2 8}$ & $-0.346(-0.876-0.184)$ & 0.201 \\
Current respiratory symptoms & $-0.163(-0.519-0.193)$ & 0.370 & $-0.051(-0.531-0.428)$ & 0.834 & $0.054(-0.419-0.528)$ & 0.822 \\
Neutrophil count & $-\mathbf{0 . 0 7 3 ( - 0 . 1 1 9 - - 0 . 0 2 7 )}$ & $\mathbf{0 . 0 0 2}$ & $0.021(-0.027-0.070)$ & 0.381 & $0.026(-0.022-0.075)$ & 0.291 \\
Presence of neutrophil elastase & $-0.091(-0405-0.224)$ & 0.572 & $0.038(-0.272-0.348)$ & 0.809 & $-0.018(-0.335-0.298)$ & 0.909 \\
IL-8 count & $-\mathbf{0 . 0 7 8 ( - 0 . 1 3 7 - - 0 . 0 1 9 )}$ & $\mathbf{0 . 0 1 0}$ & $0.016(-0.045-0.076)$ & 0.611 & $0.015(-0.045-0.076)$ & 0.618 \\
Significant infection & $-0.166(-0.522-0.189)$ & 0.359 & $0.101(-0.249-0.452)$ & 0.572 & $-0.020(-0.365-0.327)$ & 0.913 \\
Pro-inflammatory pathogens & $-0.274(-0.633-0.083)$ & 0.132 & $0.048(-0.279-0.375)$ & 0.774 & $-0.027(-0.364-0.311)$ & 0.877 \\
$\quad$ Staphylococcus aureus & $-0.231(-0.722-0.259)$ & 0.355 & $0.173(-0.262-0.618)$ & 0.435 & $-0.086(-0.574-0.401)$ & 0.728 \\
$\quad$ Haemophilus influenzae & $-\mathbf{0 . 6 4 3 ( - 1 . 1 4 3 - - 0 . 1 4 2 )}$ & $\mathbf{0 . 0 1 2}$ & $\mathbf{0 . 4 7 5}(\mathbf{0 . 0 1 2 - 0 . 9 3 7 )}$ & $\mathbf{0 . 0 4 4}$ & $0.407(-0.125-0.940)$ & 0.126 \\
$\quad$ Pseudomonas aeruginosa & $-0.343(-0.818-0.132)$ & 0.157 & $-0.483(-0.995-0.030)$ & 0.065 & $-0.316(-0.902-0.270)$ & 0.290 \\
$\quad$ Aspergillus spp. & $-0.066(-0.573-0.440)$ & 0.797 & $0.372(-0.115-0.860)$ & 0.134 & $0.284(-0.266-0.834)$ & 0.312 \\
Multiple infection & $-0.124(-0.574-0.327)$ & 0.590 & $0.035(-0.430-0.500)$ & 0.882 & $-0.109(-0.512-0.293)$ & 0.594 \\
Bronchiectasis score & $-0.025(-0.076-0.027)$ & 0.341 & $-0.025(-0.077-0.027)$ & 0.341 & $0.003(-0.048-0.054)$ & 0.911 \\
Air-trapping score & $-\mathbf{0 . 1 0 4 ( - 0 . 1 9 3 - 0 . 0 1 6 )}$ & $\mathbf{0 . 0 2 1}$ & $-0.014(-0.106-0.077)$ & 0.757 & $0.008(-0.083-0.099)$ & 0.863
\end{tabular}

Data are presented as mean ( $95 \% \mathrm{Cl}$ ) difference in $\mathrm{z}$-score between children with $\mathrm{CF}$ described by the variable compared with those not described by the variable, unless otherwise stated. Statistically significant $(p<0.05)$ changes are shown in bold. Xrs8: respiratory reactance at $8 \mathrm{~Hz}$; fres: resonant frequency of respiratory reactance; $A x$ : area under the reactance curve; IL: interleukin.

annual review, but longitudinally presence of bronchiectasis was associated with a small decrease in $f_{\text {dep }}$ $(-0.007,-0.014--0.001 ; \mathrm{p}=0.036)$.

Children with CF who had a severe genotype had lower fres $\mathrm{z}$-scores $(-0.560,-1.128--0.065 ; \mathrm{p}=0.028)$ compared with those with a mild genotype, but all other FOT outcomes were the same; longitudinally, having a severe genotype was not associated with worsening of FOT over time. Children with current respiratory symptoms had the same FOT outcomes as asymptomatic children at annual review, but respiratory symptoms were associated with an increase in Ax z-score (0.836, 0.022-1.651; $\mathrm{p}=0.044)$ over 1 year.

\section{Discussion}

In this large cohort study we found that the FOT was a relatively insensitive tool to detect underlying lung disease in young children with CF. In general, children with CF who had pronounced respiratory disease, including free neutrophil elastase activity, infection with pro-inflammatory pathogens or structural lung abnormalities had similar FOT outcomes to those children without detectable lung disease. In addition, the detection of CF lung disease using gold-standard surveillance outcomes of BAL and chest CT was not associated with worsening FOT outcomes over 1 year. Our study suggests that the FOT may not be a suitable tool for the surveillance of lung disease in young children with CF.

TABLE 4 Longitudinal analysis of respiratory resistance outcomes and cystic fibrosis (CF) lung disease parameters

\begin{tabular}{|c|c|c|c|c|c|}
\hline & Subjects & Change in Rrs8 z-score & p-value & Change in fdep & $\mathrm{p}$-value \\
\hline Current respiratory symptoms & 16 & $0.442(-0.061-0.946)$ & 0.085 & $-0.029(-0.093-0.034)$ & 0.366 \\
\hline Presence of neutrophil elastase & 61 & $-0.083(-0.373-0.208)$ & 0.577 & $0.004(-0.033-0.041)$ & 0.841 \\
\hline IL-8 count & 180 & $-0.032(-0.088-0.024)$ & 0.266 & $0.006(-0.001-0.012)$ & 0.119 \\
\hline Significant infection & 90 & $0.081(-0.272-0.434)$ & 0.654 & $-0.009(-0.050-0.033)$ & 0.687 \\
\hline Pro-inflammatory pathogen & 74 & $0.238(-0.061-0.536)$ & 0.119 & $-0.015(-0.041-0.058)$ & 0.734 \\
\hline Haemophilus influenzae & 21 & $0.371(-0.183-0.924)$ & 0.189 & $-0.046(-0.100-0.007)$ & 0.089 \\
\hline Pseudomonas aeruginosa & 20 & $0.046(-0.503-0.594)$ & 0.870 & $-0.030(-0.087-0.028)$ & 0.103 \\
\hline Aspergillus spp. & 19 & $0.332(-0.211-0.875)$ & 0.230 & $-0.033(-0.087-0.022)$ & 0.239 \\
\hline Multiple infection & 43 & $0.237(-0.126-0.318)$ & 0.156 & $-0.029(-0.061-0.004)$ & 0.086 \\
\hline Bronchiectasis score & 93 & $-0.001(-0.055,0.055)$ & 0.998 & $-0.007(-0.014--0.001)$ & 0.036 \\
\hline Air-trapping score & 47 & $0.037(-0.074-0.147)$ & 0.517 & $-0.017(-0.030--0.005)$ & 0.008 \\
\hline
\end{tabular}

Data are presented as $\mathrm{n}$ or mean $(95 \% \mathrm{CI})$ change over 1 year in children with $\mathrm{CF}$ described by the variable compared with those not described by the variable, unless stated otherwise. Statistically significant $(p<0.05)$ changes are shown in bold. Rrs8: respiratory resistance at $8 \mathrm{~Hz}$; $f$ dep: frequency dependence of respiratory resistance; IL: interleukin. 
TABLE 5 Longitudinal analysis of respiratory reactance outcomes and cystic fibrosis (CF) lung disease parameters

\begin{tabular}{|c|c|c|c|c|c|c|c|}
\hline & Subjects & $\begin{array}{l}\text { Change in } X_{\text {rs8 }} \\
z \text {-score }\end{array}$ & p-value & Change in fres z-score & p-value & $\begin{array}{c}\text { Change in } A x \\
z \text {-score }\end{array}$ & p-value \\
\hline Severe genotype & 92 & $-0.014(-0.355-0.326)$ & 0.934 & $-0.322(-0.718-0.074)$ & 0.111 & $-0.033(-0.423-0.358)$ & 0.870 \\
\hline Neutrophil count & 179 & $0.037(-0.034-0.108)$ & 0.310 & $0.091(0.001-0.162)$ & 0.022 & $-0.003(-0.071-0.092)$ & 0.921 \\
\hline $\begin{array}{l}\text { Presence of neutrophil } \\
\text { elastase }\end{array}$ & 61 & $-0.018(-0.449-0.413)$ & 0.934 & $0.380(-0.122-0.881)$ & 0.138 & $0.121(-0.387-0.629)$ & 0.641 \\
\hline Significant infection & 90 & $-0.074(-0.459-0.607)$ & 0.786 & $-0.127(-0.687-0.432)$ & 0.655 & $0.174(-0.381-0.728)$ & 0.540 \\
\hline $\begin{array}{l}\text { Pro-inflammatory } \\
\text { pathogen }\end{array}$ & 74 & $-0.270(-0.706-0.166)$ & 0.224 & $-0.269(-0.939-0.401)$ & 0.432 & $0.042(-0.675,0.591)$ & 0.896 \\
\hline Staphylococcus aureus & 28 & $-0.167(-0.909-0.575)$ & 0.659 & $0.103(-0.544-0.750)$ & 0.756 & $0.441(-0.364-1.246)$ & 0.283 \\
\hline Haemophilus influenzae & 21 & $-0.227(-1.051-0.596)$ & 0.588 & $-0.360(-0.415-1.136)$ & 0.362 & $1.087(0.261-1.913)$ & 0.010 \\
\hline Pseudomonas aeruginosa & 20 & $-0.268(-1.050-0.514)$ & 0.502 & $-0.010(-0.730-0.711)$ & 0.979 & $0.202(-0.597-1.000)$ & 0.621 \\
\hline
\end{tabular}

Data are presented as $\mathrm{n}$ or mean $(95 \% \mathrm{CI})$ change in $\mathrm{z}$-score over 1 year in children with $\mathrm{CF}$ described by the variable compared with those not described by the variable, unless stated otherwise. Statistically significant $(p<0.05)$ changes are shown in bold. Xrs8: respiratory reactance at $8 \mathrm{~Hz}$; fres: resonant frequency of respiratory reactance; $A x$ : area under the reactance curve; IL: interleukin.

There are a small number of studies that have examined the ability of the FOT to detect lung disease in children with CF. A previous study from the AREST CF early surveillance programme provided data from 24 children (aged 0-6 years) with CF who had low-frequency FOT performed under general anaesthetic prior to the collection of BAL fluid [8]. In this study there were significant associations between tissue mechanics (tissue damping and hysteresivity) and markers of pulmonary inflammation including total cell count, neutrophil count and IL-8; however, there were no associations found between FOT outcomes and pulmonary infection status [8]. The only other study to examine FOT outcomes and pulmonary infection in young children examined respiratory resistance and reactance at $5 \mathrm{~Hz}$ using the impulse oscillation technique in 30 preschool children (aged 2-7 years) with CF and found no associations between oscillatory mechanics and upper airway infection status [19]. In a small number of children aged $>7$ years, resistance was lower and reactance less negative after treatment for a respiratory exacerbation [35]. Other studies have shown that a small proportion of children with CF have abnormal respiratory resistance and reactance values compared with healthy reference data $[24,25]$, and children with CF who have current respiratory symptoms have higher resistance and lower reactance compared with asymptomatic children with CF [24].

The present study is the first to comprehensively examine the ability of the FOT to detect underlying pulmonary inflammation and infection in a large cohort of preschool children with CF. In general we found that FOT outcomes were insensitive to detect underlying lung disease when examined either at one point in time, or longitudinally over 1 year. We found that the $f_{\text {dep }}$ and $X_{\mathrm{rs}} 8$ were the most sensitive outcomes of FOT in our cohort, which may reflect an increase in the resistance of the peripheral airways and/or an increase in ventilation inhomogeneity of the lung [36, 37]. Both of these outcomes were associated with the number of neutrophils, IL-8 concentrations, presence of $H$. influenzae and air trapping score, although the magnitudes of the effect sizes were small and were most prominent in the cross-sectional rather than the longitudinal analysis. It is important to note that the children in this study were clinically well enough to receive a general anaesthetic at the time of their lung function assessment, and therefore the children with exacerbated respiratory symptoms who we would expect to have the greatest decline in lung function were excluded from the analysis. In addition, as there have been no studies that have examined the natural variability of the FOT in clinically stable young children with CF, it is not known what clinically significant changes in respiratory resistance or reactance outcomes are in this population. The present study suggests that the FOT technique may be insensitive to the presence or progression of respiratory inflammation, infection and structural disease in early CF lung disease.

It is unclear which lung function tests are the most appropriate for the surveillance of preschool children with CF. While spirometry is the most common tool to evaluate respiratory function in adults and children aged $>6$ years, it is not a reliable method to assess lung function in younger children due to the cooperation and coordination required to perform forced expiratory manoeuvres. In addition, the majority 
of children with CF have spirometry outcomes within the healthy reference range until adolescence [38]. The multiple breath washout (MBW) test may be a more suitable tool for the surveillance of preschool children with CF. As a tidal breathing test, MBW is easier to perform than spirometry [15]. The lung clearance index (LCI) from the MBW test is the first index to decline in preschool children with CF and is more sensitive than forced expiratory volume in $1 \mathrm{~s}$ to detect underlying lung disease $[15,16,39]$. Further research is needed to evaluate the ability of the LCI to detect lower respiratory tract inflammation, infection or structural disease in children with CF aged 3-6 years.

Infection with $H$. influenzae seems to adversely affect lung function in young children with CF. While we found no associations between the presence of any respiratory pathogen and FOT outcomes, when we examined the presence of pathogens individually we found that infection with $H$. influenzae was associated with increased $R \mathrm{rs}$ at $8 \mathrm{~Hz}$ and resonant frequency, and reduced $X \mathrm{rs} 8$ and $f$ dep in the cross-sectional analysis, and increased $A \mathrm{x}$ in the longitudinal analysis. We found no associations between the presence of any other individual pathogens and FOT outcomes. A previous study by the AREST CF group showed that the presence of $H$. influenzae in the first 2 years of life was associated with persistently lower forced expiratory volume in $0.75 \mathrm{~s}$ and forced vital capacity up to school age [40] and increased LCI in infancy [41]. In addition, BRUMBACK et al. [42] found that recent $H$. influenzae infection was associated with lower forced vital capacity in preschool children with CF. Infection with $H$. influenzae has also been associated with pulmonary inflammation [14,31] and pulmonary exacerbations [43] in children with CF. These data indicate that $H$. influenzae is highly pathogenic in young children with $\mathrm{CF}$, and should be targeted for eradication and treatment to improve lung function outcomes.

A major strength of the study is the large population of preschool children with matched FOT and clinical surveillance outcomes. Other strengths include the use of BAL fluid for quantification of lower respiratory tract inflammation and pathogens, and chest CT for the quantification of structural lung disease. Serial measurements of FOT during childhood allowed for a longitudinal analysis of change in FOT outcomes over time. One limitation of the use of annual surveillance data is that it does not provide information on the clinical status of the child between annual visits. These data were collected when the children were relatively well rather than during an exacerbation, and therefore do not provide insight into the role of exacerbations on lung function outcomes. It is also unclear which markers of pulmonary inflammation are the most suitable in paediatric CF lung disease. We found small but statistically significant associations between neutrophil count and IL-8 levels and respiratory impedance outcomes, but found no relationship between neutrophil elastase activity and FOT outcomes.

We performed a comprehensive assessment of the ability of the FOT to detect the presence and progression of lung disease in young children with CF. Our results indicate that outcomes of respiratory resistance and reactance from the FOT are not associated with the presence or progression of pulmonary inflammation, lower respiratory tract pathogens or structural lung disease in children with CF aged 3-6 years when assessed using annual surveillance data. However, FOT may still have value in improving our understanding of the physiological changes associated with early CF lung disease. Further insights into the utility of the FOT in early CF lung disease, especially their ability to detect and monitor pulmonary exacerbations, are needed to determine whether FOT has any clinical role as a lung function tool in CF.

\section{Acknowledgements}

We would like to thank the respiratory fellows who performed the bronchoscopies and laboratory staff members who processed and analysed the samples. We would also like to thank the participants and their families who contribute to the AREST CF programme.

\section{References}

1 Sly PD, Brennan S, Gangell C, et al. Lung disease at diagnosis in infants with cystic fibrosis detected by newborn screening. Am J Respir Crit Care Med 2009; 180: 146-152.

2 Stick SM, Brennan S, Murray C, et al. Bronchiectasis in infants and preschool children diagnosed with cystic fibrosis after newborn screening. J Pediatr 2009; 155: 623-628.

3 Ramsey KA, Ranganathan S. Interpretation of lung function in infants and young children with cystic fibrosis. Respirology 2014; 19: 792-799.

4 Zemanick ET, Hoffman L, Rosenfeld M. Narrowing in on early cystic fibrosis lung disease. Am J Respir Crit Care Med 2014; 190: 1082-1084.

5 Dakin CJ, Pereira JK, Henry RL, et al. Relationship between sputum inflammatory markers, lung function, and lung pathology on high-resolution computed tomography in children with cystic fibrosis. Pediatr Pulmonol 2002; 33: $475-482$.

6 Mayer-Hamblett N, Aitken ML, Accurso FJ, et al. Association between pulmonary function and sputum biomarkers in cystic fibrosis. Am J Respir Crit Care Med 2007; 175: 822-828.

7 Sagel SD, Sontag MK, Wagener JS, et al. Induced sputum inflammatory measures correlate with lung function in children with cystic fibrosis. J Pediatr 2002; 141: 811-817.

8 Brennan S, Hall GL, Horak F, et al. Correlation of forced oscillation technique in preschool children with cystic fibrosis with pulmonary inflammation. Thorax 2005; 60: 159-163. 
9 Dakin $\mathrm{CJ}$, Numa $\mathrm{AH}$, Wang $\mathrm{H}$, et al. Inflammation, infection, and pulmonary function in infants and young children with cystic fibrosis. Am J Respir Crit Care Med 2002; 165: 904-910.

10 Linnane BM, Hall GL, Nolan G, et al. Lung function in infants with cystic fibrosis diagnosed by newborn screening. Am J Respir Crit Care Med 2008; 178: 1238-1244.

11 Nixon GM, Armstrong DS, Carzino R, et al. Early airway infection, inflammation, and lung function in cystic fibrosis. Arch Dis Child 2002; 87: 306-311.

12 Peterson-Carmichael SL, Harris WT, Goel R, et al. Association of lower airway inflammation with physiologic findings in young children with cystic fibrosis. Pediatr Pulmonol 2009; 44: 503-511.

13 Pillarisetti N, Williamson E, Linnane B, et al. Infection, inflammation, and lung function decline in infants with cystic fibrosis. Am J Respir Crit Care Med 2011; 184: 75-81.

14 Rosenfeld M, Gibson RL, McNamara S, et al. Early pulmonary infection, inflammation, and clinical outcomes in infants with cystic fibrosis. Pediatr Pulmonol 2001; 32: 356-366.

15 Aurora P, Bush A, Gustafsson P, et al. Multiple-breath washout as a marker of lung disease in preschool children with cystic fibrosis. Am J Respir Crit Care Med 2005; 171: 249-256.

16 Aurora P, Stanojevic S, Wade A, et al. Lung clearance index at 4 years predicts subsequent lung function in children with cystic fibrosis. Am J Respir Crit Care Med 2011; 183: 752-758.

17 Calogero C, Simpson SJ, Lombardi E, et al. Respiratory impedance and bronchodilator responsiveness in healthy children aged 2-13 years. Pediatr Pulmonol 2013; 48: 707-715.

18 Kozlowska WJ, Bush A, Wade A, et al. Lung function from infancy to the preschool years after clinical diagnosis of cystic fibrosis. Am J Respir Crit Care Med 2008; 178: 42-49.

19 Nielsen KG, Pressler T, Klug B, et al. Serial lung function and responsiveness in cystic fibrosis during early childhood. Am J Respir Crit Care Med 2004; 169: 1209-1216.

20 Vilozni D, Bentur L, Efrati O, et al. Spirometry in early childhood in cystic fibrosis patients. Chest 2007; 131: 356-361.

21 Hall GL, Sly PD, Fukushima T, et al. Respiratory function in healthy young children using forced oscillations. Thorax 2007; 62: 521-526.

22 Udomittipong K, Sly PD, Patterson HJ, et al. Forced oscillations in the clinical setting in young children with neonatal lung disease. Eur Respir J 2008; 31: 1292-1299.

23 Lebecque P, Stănescu D. Respiratory resistance by the forced oscillation technique in asthmatic children and cystic fibrosis patients. Eur Respir J 1997; 10: 891-895.

24 Gangell CL, Horak F Jr, Patterson HJ, et al. Respiratory impedance in children with cystic fibrosis using forced oscillations in clinic. Eur Respir J 2007; 30: 892-897.

25 Solymar L, Aronsson PH, Sixt R. The forced oscillation technique in children with respiratory disease. Pediatr Pulmonol 1985; 1: 256-261.

26 Duiverman EJ, Den Boer JA, Roorda RJ, et al. Lung function and bronchial responsiveness measured by forced oscillometry after bronchopulmonary dysplasia. Arch Dis Child 1988; 63: 727-732.

27 Vrijlandt EJ, Boezen HM, Gerritsen J, et al. Respiratory health in prematurely born preschool children with and without bronchopulmonary dysplasia. J Pediatr 2007; 150: 256-261.

28 Rosenfeld M, Allen J, Arets BH, et al. An official American Thoracic Society workshop report: optimal lung function tests for monitoring cystic fibrosis, bronchopulmonary dysplasia, and recurrent wheezing in children less than 6 years of age. Ann Am Thorac Soc 2013; 10: S1-S11.

29 Bodini A, D'Orazio C, Peroni D, et al. Biomarkers of neutrophilic inflammation in exhaled air of cystic fibrosis children with bacterial airway infections. Pediatr Pulmonol 2005; 40: 494-499.

30 Beydon N, Davis SD, Lombardi E, et al. An official American Thoracic Society/European Respiratory Society statement: pulmonary function testing in preschool children. Am J Respir Crit Care Med 2007; 175: 1304-1345.

31 Gangell C, Gard S, Douglas T, et al. Inflammatory responses to individual microorganisms in the lungs of children with cystic fibrosis. Clin Infect Dis 2011; 53: 425-432.

32 Mott LS, Park J, Murray CP, et al. Progression of early structural lung disease in young children with cystic fibrosis assessed using CT. Thorax 2012; 67: 509-516.

33 de Onis M, Onyango A, Borghi E, et al. Development of a WHO growth reference for school-aged children and adolescents. Bull World Health Organ 2007; 85: 660-667.

34 WHO Multicentre Growth Reference Study Group. WHO Child Growth Standards based on length/height, weight and age. Acta Paediatr Suppl 2006; 450: 76-85.

35 Ren CL, Brucker JL, Rovitelli AK, et al. Changes in lung function measured by spirometry and the forced oscillation technique in cystic fibrosis patients undergoing treatment for respiratory tract exacerbation. Pediatr Pulmonol 2006; 41: 345-349.

36 Frey U. Forced oscillation technique in infants and young children. Paediatr Respir Rev 2005; 6: 246-254.

37 Oostveen E, MacLeod D, Lorino H, et al. The forced oscillation technique in clinical practice: methodology, recommendations and future developments. Eur Respir J 2003; 22: 1026-1041.

38 Marostica PJ, Weist AD, Eigen H, et al. Spirometry in 3- to 6-year-old children with cystic fibrosis. Am J Respir Crit Care Med 2002; 166: 67-71.

39 Owens CM, Aurora P, Stanojevic S, et al. Lung Clearance Index and HRCT are complementary markers of lung abnormalities in young children with CF. Thorax 2011; 66: 481-488.

40 Ramsey KA, Ranganathan S, Park J, et al. Early respiratory infection is associated with reduced spirometry in children with cystic fibrosis. Am J Respir Crit Care Med 2014; 190: 1111-1116.

41 Simpson SJ, Ranganathan S, Park J, et al. Progressive ventilation inhomogeneity in infants with cystic fibrosis after pulmonary infection. Eur Respir J 2015; 46: 1680-1690.

42 Brumback LC, Davis SD, Kerby GS, et al. Lung function from infancy to preschool in a cohort of children with cystic fibrosis. Eur Respir J 2013; 41: 60-66.

43 Rayner RJ, Hiller EJ, Ispahani P, et al. Haemophilus infection in cystic fibrosis. Arch Dis Child 1990; 65: 255-258. 